\title{
Formatação dos processos judiciais que demandam medicamentos no Município de João Pessoa-PB, 2009-2012
}

Formatting of lawsuits demanding drugs in Município de João Pessoa-PB, 2009-2012

\section{Rildeane Alves Gabriel}

Estudante, Graduanda. Universidade Federal da Paraíba. Itabaiana, Brasil.

\section{Sabine Helena Dantas}

Estudante, Graduanda. Universidade Federal da Paraíba. João Pessoa, Brasil.

\section{Rossana Maria Souto Maior Serrano}

Professora, Doutora. Universidade Federal da Paraíba. João Pessoa, Brasil.

Resumo: Esta pesquisa é parte do projeto SIMSUS - Sistema de Informação sobre Medicamentos do Sistema Único de Saúde: acessibilidade e uso racional. Faz-se uma análise dos elementos que compõem os processos judiciais que demandam aquisição de medicamentos no município de João Pessoa. A pesquisa foi realizada na Gerencia de Medicamentos do Município de João Pessoa (GEMAF); foram analisados todos os processos judiciais que demandavam a aquisição de medicamentos nos anos de 2009 a 2012. Para a coleta de dados foi estruturado um roteiro com questões referentes a: perfil do usuário (idade, sexo, região onde reside) e perfil do processo judicial (documentos que o compunham e existência, nos autos, de laudo médico, exames, o código da Classificação Internacional de Doenças (CID)). As informações foram sistematizadas em razão da composição do processo judicial e da natureza farmacológica do produto solicitado. Foi constatada a falta de um padrão na estruturação dos processos e a ausência frequente de informações fundamentais para análise da pertinência farmacológica do medicamento. Evidenciou-se que grande parte dos processos não trazia informações sobre a idade do usuário, a CID ou a duração do tratamento. Concluiu-se pela necessidade de articulação entre o Gestor Municipal e o Poder Judiciário sobre a padronização das informações dos processos judiciais, nos aspectos formais de composição do processo.

Palavras-chave: Judicialização; assistência farmacêutica; acessibilidade.

Key-words: Legalization; pharmaceutical care; accessibility

\section{Introdução}

A discussão sobre o uso racional de medicamentos não é algo novo no Brasil. Desde o inicio da década de 1990 essa temática tem sido objeto de conceituações e 
normatizações por parte do Ministério da Saúde, fundamentada nas recomendações da Organização Mundial da Saúde.

Como base no princípio constitucional da gratuidade da assistência da saúde e das novas políticas no campo da assistência farmacêutica, o SUS vem se organizando em programas para oferecer medicamentos para a população, quais sejam: o Programa de Farmácia Básica, que dispõe de uma lista de mais de 450 medicamentos para atender a 90\% das demandas da atenção básica de saúde, e o Programa dos Componentes Especiais da Assistência Farmacêutica, composto pelos programas dos componentes estratégicos, e dos componentes excepcionais. Dentro de cada um desses programas cada instância de governo tem competências definidas em lei e participa do processo de financiamento.

Entretanto, mesmo com a diversidade de programas de assistência farmacêutica, não são raras queixas e denúncias sobre a falta de medicamento e a dificuldade de acesso ao mesmo no serviço público, o que tem demandado a busca do Ministério Público ou do Poder Judiciário para a garantia do direito de acesso. Tal busca tem se constituído, em todos os Estados da federação, um problema diante dos custos que essas demandas têm gerado. Esse fato tem sido definido como um fenômeno, que passou a ser denominado de Judicialização da Saúde.

Alguns problemas vêm se configurando a partir da operacionalidade dessas demandas judiciais, quais sejam: a descaracterização da padronização dos medicamentos no serviço público, uma vez que prescritores indicam medicamentos fora dessa padronização, mesmo quando existem outros de indicação terapêutica semelhante disponível na rede; a prescrição de medicamentos por nome de marca, sendo que existe o genérico disponível; e o uso do recurso judicial para solicitar medicamento que poderiam ser solicitados de forma rotineira ou pela via administrativa.

Este estudo analisa as demandas judiciais por medicamentos do período de 2009 a 2012 no município de João Pessoa, para subsidiar a análise da realidade desse município que possa sinalizar para uma melhor racionalização no atendimento dessas demandas pelo poder judiciário e pela Secretaria de Saúde Municipal.

O estudo parte dos pressupostos de que o medicamento é um direito social inquestionável e de que, sem dúvidas, é papel do Estado garantir a assistência farmacêutica de qualidade e de forma gratuita a todos os cidadãos brasileiros. 
Entretanto, esse direito está vinculado ao uso racional desses produtos uma vez que medicamento não é um bem de consumo livre, na medida em que seu uso apresenta riscos para a saúde, se utilizado fora dos padrões orientados.

O uso racional de medicamentos constitui-se num processo que passa por uma prescrição pertinente do ponto de vista terapêutico; pela disponibilidade do produto no tempo certo; pela dispensação com as devidas orientações farmacêuticas e pela análise da dose, tempo de tratamento e forma de uso; e, por fim, pela administração correta.

Essas etapas, que são parte do ciclo da assistência farmacêutica preconizado pelo Ministério da Saúde, constituíram os fundamentos da análise dos processos judiciais, uma vez que tem sido crescente o aumento dessas demandas, e os produtos medicamentosos solicitados muitas vezes fogem ao processo de padronização adotado, o que gera compras sem licitação, ou compras de produtos de custo maior para igual ação terapêutica.

\section{Metodologia}

Trata-se de um estudo quali-quantitativo, de natureza descritiva, onde foram analisados todos os processos judiciais demandados contra o município de João Pessoa, Estado da Paraíba, no período de 2009 a 2012.

A coleta de dados partiu de um questionário estruturado e testado, cujas variáveis trabalhadas buscaram identificar o perfil do usuário quanto à idade, sexo, região onde reside; e o perfil do processo judicial, em relação à vara, documentos que compõem os autos, prazo de atendimento determinado em sentença, existência, no processo judicial, de laudo médico, exames, descrição da CID.

As informações foram sistematizadas em razão da composição do processo judicial e da natureza farmacológica do produto solicitado. Os dados foram sistematizados em planilha Excel e analisados a luz das políticas públicas implementadas.

\section{Resultados}

\section{Dados gerais dos processos e dos produtos}

Foram analisados 159 processos judiciais atendidos pela Gerencia de Medicamentos da Secretaria Municipal de Saúde de João Pessoa (GEMAF/SMS), do 
conjunto de demandas judiciais para a Secretaria de Saúde do município nos anos de 2009 a 2012.

Foram excluídas do estudo as demandas judiciais por procedimentos médicos e serviços e tratou-se apenas daquelas que demandaram produtos farmacêuticos e correlatos (Gráfico 1).

Gráfico 1 - Processos judiciais e produtos e insumos demandados a Secretaria de Saúde do município de João Pessoa, segundo o ano. 2009-2012.

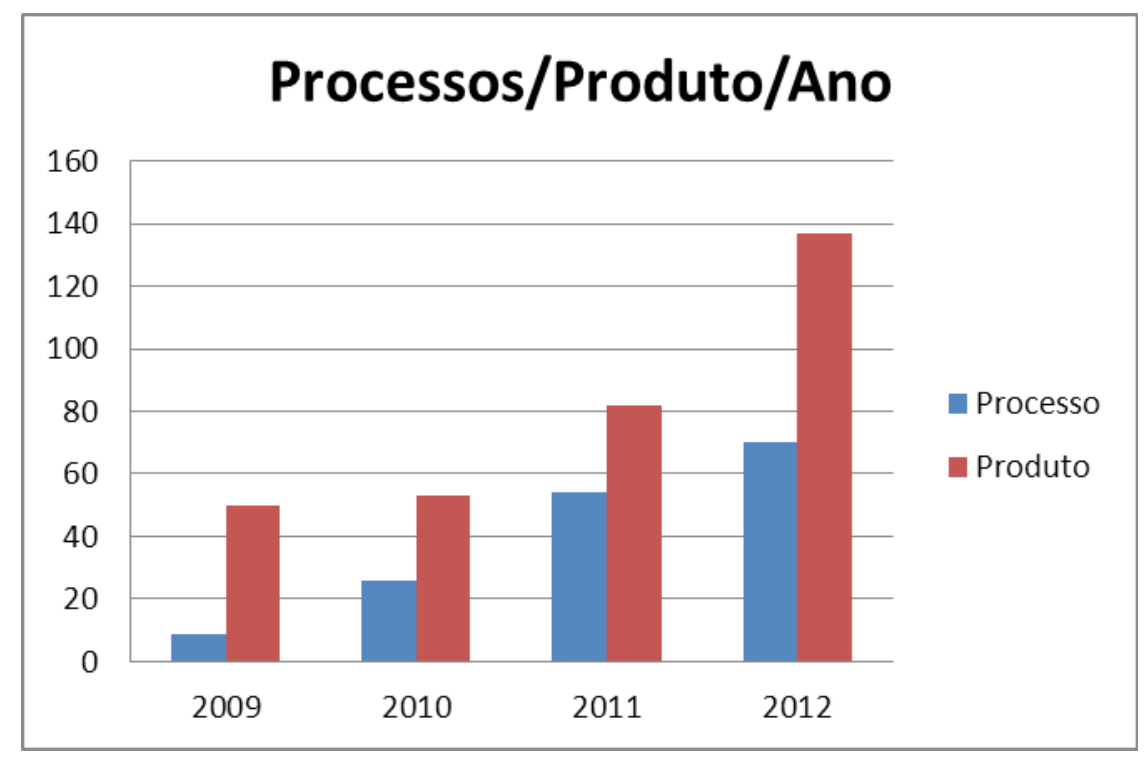

Fonte: GEMAF/SMS João Pessoa. Elaborado pelas autoras.

O estudo analisou as demandas por medicamentos, dietas e insumos, entendendo por este último, produtos como fraldas, kits reagentes de teste rápido, sondas e outros de apoio terapêutico.

Os dados obtidos apontam para uma expressiva procura por medicamentos (Tabela 1).

Tabela 1 - Demandas judiciais segundo o produto demandado, por ano. Município de João Pessoa, 2009-2012.

\begin{tabular}{|c|c|c|c|c|c|c|}
\hline \multirow[t]{2}{*}{ ProdutolAno } & \multicolumn{2}{|c|}{2009} & 2010 & 2011 & 2012 & \multirow[t]{2}{*}{ Total } \\
\hline & & $\%$ & No $\%$ & No $\%$ & No $\%$ & \\
\hline Dietas & - & - & 1222 & 1012 & 2619 & 48 \\
\hline Insumos & 11 & 22 & 1019 & 78 & 3123 & 59 \\
\hline Medicamentos & 39 & 78 & 3158 & 6579 & 8058 & 206 \\
\hline Total & 50 & & 53 & 82 & 137 & \\
\hline
\end{tabular}

Fonte: GEMAF/SMS João Pessoa. Elaborado pelas autoras. 
Tal informação configura-se como um elemento a ser oportunamente investigado, considerando que a rede dispõe de um amplo arsenal terapêutico no Programa de Farmácia Básica e, ainda, que existem programas de componentes especializados e excepcionais sob a responsabilidade de outras esferas de governo.

\section{Perfil dos usuários}

Dos 159 processos avaliados no tocante ao sexo do usuário, identificou-se que 86 (54\%) eram do sexo feminino e 73 do sexo masculino, o que não representa expressiva diferença, mas que reproduz o fato de que as mulheres vão mais ao médico (Tabela 2).

Tabela 2 - Autores das ações segundo o sexo e o ano da ação. Município de João Pessoa, 2009-2012

\begin{tabular}{ccccc}
\hline Ano & \multicolumn{2}{c}{ Masculino } & \multicolumn{2}{c}{ Feminino } \\
& No & $\%$ & $\mathbf{N}^{\circ}$ & $\%$ \\
\hline 2009 & 7 & 78 & 2 & 22 \\
2010 & 12 & 46 & 14 & 54 \\
2011 & 17 & 32 & 36 & 68 \\
2012 & 32 & 48 & 35 & 52 \\
\hline
\end{tabular}

Fonte: GEMAF/SMS João Pessoa. Elaborado pelas autoras.

No tocante à idade dos pleiteantes, observou-se a maior incidência dos usuários maiores de 66 anos, seguidos dos que estão na faixa etária entre 37 e 56 anos. $18 \%$ dos usuários estavam na faixa etária de menor de um ano. Nenhum usuário estava na faixa etária de 16 a 25 anos (Gráfico 2).

Gráfico 2 - Autores das ações segundo a idade. Município de João Pessoa, 2009-2012.

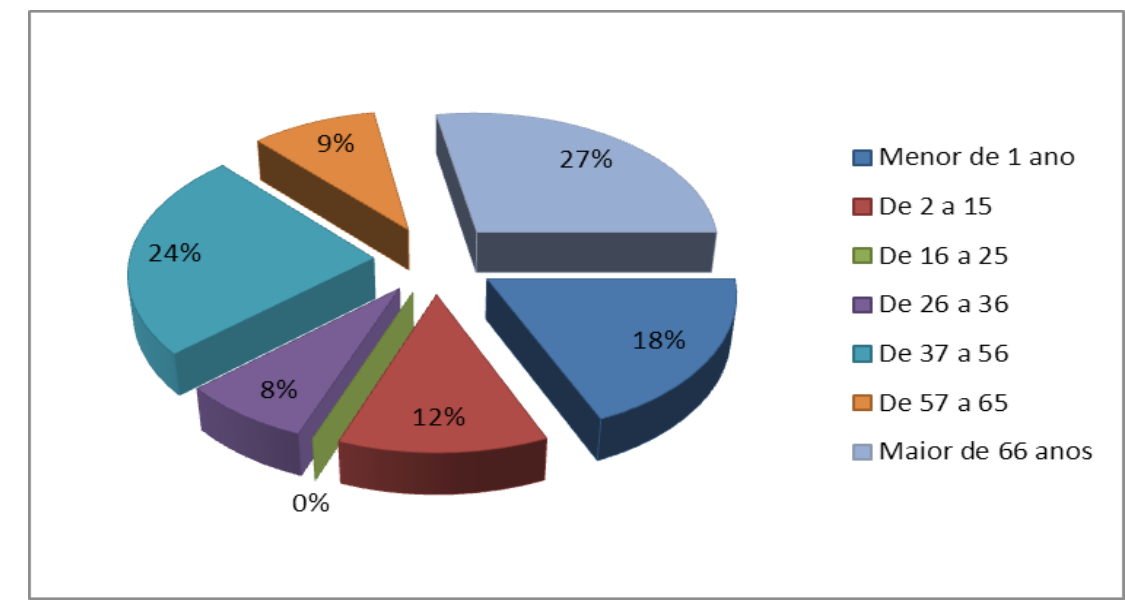

Fonte: GEMAF/SMS João Pessoa. Elaborado pelas autoras. 
A grande maioria dos processos trazia o endereço do usuário, com exceção de 61 deles, que não apresentavam essa informação na petição inicial, o que dificulta o contato do serviço com o demandante para a entrega do produto.

O perfil de origem geográfica dos usuários foi observado na tentativa de se analisar a relação entre poder aquisitivo/demanda judicial. Como os processos não são instruídos com outra informação que nos possibilite melhor analise, adotou-se o bairro em que o usuário reside como forma de mapear as demandas no território e quanto a esse aspecto.

Observou-se, quanto a isso, um perfil muito diversificado, sendo a maior parte dos processos decorrentes de moradores de bairros de classe média e média baixa. Esse fato desmistifica a impressão de que as demandas judiciais são, em sua maioria, originárias de usuários de maior poder aquisitivo.

\section{Formatação dos processos}

Compreendendo que a formatação do processo judicial é fundamental para a análise do mérito da pertinência terapêutica, apontamos, em discussão e análise prévia, alguns elementos fundamentais a sua composição, quais sejam:

1. nome e endereço do usuário;

2. cópia do cartão SUS (documento de identificação nacional do usuário);

3. prescrição médica;

4. laudo médico com a identificação do Código Internacional de Doenças;

De forma complementar, foram considerados oportunos para análise farmacológica e para o melhor controle da gestão do serviço:

5. exames comprobatórios da patologia (caso necessário);

6. duração do tratamento (requisito legal de uma prescrição);

7. pertinência do prescritor para a especialidade prescrita.

$\mathrm{Na}$ análise dos processos e aplicando o questionário estruturado com os itens acima descritos observamos que:

- $49 \%$ dos processos apresentavam a CID;

- $\quad 43 \%$ estavam acompanhados de laudos de médicos ou de outros profissionais;

- $11 \%$ apresentavam cópia de exames laboratoriais ou de imagem;

- $35 \%$ das prescrições especificavam a duração do tratamento; 
- $28 \%$ dos processos apresentavam cópia do cartão SUS;

- $\quad$ em $47 \%$ dos processos havia pertinência entre o prescritor e o produto prescrito ou seja, quem prescrevia estava identificado como um especialista na área (Tabela 3).

Tabela 3 - Presença (\%) de elementos presentes nos autos na petição inicial, segundo o ano da inicial. Município de João Pessoa, 2009-2012.

\begin{tabular}{l|cccc|c}
\hline \multicolumn{1}{c|}{ Elemento $\backslash$ Ano } & 2009 & 2010 & 2011 & 2012 & $\%$ \\
\hline CID & 5 & 7 & 12 & 54 & 49 \\
Especialista & 4 & 11 & 18 & 42 & 47 \\
Laudo & 3 & 7 & 17 & 42 & 43 \\
Duração do tratamento & 2 & 2 & 18 & 34 & 35 \\
Cartão SUS & 1 & 3 & 10 & 31 & 28 \\
Exames & 1 & 1 & 4 & 12 & 11 \\
\hline Total de Processos & 9 & 26 & 54 & 70 & 100 \\
\hline
\end{tabular}

Fonte: GEMAF/SMS João Pessoa. Elaborado pelas autoras.

Constata-se que há necessidade de uma melhor formatação dos processos judiciais que demandam produtos farmacêuticos, de forma a possibilitar uma melhor análise farmacológica e clínica das prescrições que referende ou proponha mudanças na terapêutica demandada.

A ausência de laudos e exames, observada na maior parte dos processos, impossibilita uma contraposição fundamentada da gestão em melhor defesa do direito do usuário e do serviço; a falta de definição do tempo de tratamento impossibilita limites ao tempo da sentença proferida.

\section{Diversidade farmacológica}

$\mathrm{Na}$ análise dos produtos solicitados, observamos a demanda por produtos disponíveis na rede de atenção básica, tais como anti-hipertensivos e insulinas, bem como alguns por produtos cuja responsabilidade está pactuada para a gestão estadual. Tal fato demonstra a falta de informação dos usuários sobre os produtos disponíveis na rede e a possibilidade de acesso em serviço de rotina ou por via administrativa.

Observa-se que poucas são as prescrições com o uso da Denominação Comum Brasileira (DCB), o que inviabiliza a intercambialidade de produtos de referência pelo genérico ou vice e versa. 


\section{Considerações finais}

O direito de pedir judicialmente um medicamento ou insumo de saúde não pode ser limitado apenas pelo limite financeiro do poder público: é preciso compreender que um bem de saúde pode significar para muitos vida ou morte. Entretanto, o direito do usuário deve estar pautado pela necessidade sanitária real e pela racionalidade do seu pedido.

Cabe, hoje, aos poderes judiciário e executivo voltados à saúde, a pactuação para a melhor instrução processual, visando principalmente criar a possibilidade de diálogo com prescritores e operadores do direito.

Não é cabível que instrumentos normativos sejam violados nas petições iniciais, tais como a obrigatoriedade de prescrição pela DCB dentro do serviço público, ou a obrigatoriedade da determinação da duração do tratamento em toda prescrição.

Outro aspecto que este estudo nos aponta a necessidade de um mecanismo de informação aos usuários sobre as competências dos diversos níveis de governo, possibilitando, assim, que as demandas judiciais sejam dirigidas a quem de direito.

Por fim, destacamos que o monitoramento constante das demandas judiciais podem constituir novas realidades terapêuticas a serem cobertas por ações civis públicas ou, ainda, pela renovação das padronizações de medicamentos da rede SUS.

\section{Referências}

BRASIL. Conselho Nacional de Saúde. Política nacional de assistência farmacêutica: Resolução CNS n 338/2004. Brasília : CNS, 2004.

BRASIL. Constituição (1988). Constituição da República Federativa do Brasil. Brasília : Senado Federal, 1998.

BRASIL. Lei $n^{\circ}$ 8.080, de 19 de setembro de 1990. Dispõe sobre as condições para a promoção, proteção e recuperação da saúde, a organização e o funcionamento dos serviços correspondentes e dá outras providências. Brasília, 20 set. 1990.

BRASIL. Ministério da Saúde. Assistência farmacêutica: planejar, organizar, avaliar é preciso. Brasília : Ministério da Saúde, 2006c.

BRASIL. Ministério da Saúde. Política nacional de medicamentos: Portaria GM/MS n. ${ }^{\circ}$ 3.916, de 1998. Brasília : Ministério da Saúde, 1998.

EDAIS PEPE, V. L. et al. A Judicialização da Saúde e os Novos Desafios da Gestão da Assistência Farmacêutica. Ciência e Saúde Coletiva, Rio de Janeiro; 15(5), 2010. 
[on line]. Disponível em http://www.scielo.br/scielo.php?script=sci_arttext\&pid=S141381232010000500015, acessado em 19 jul. 2013.

CUNHA JÚNIOR, Dirley da. Curso de Direito Constitucional. Salvador : JusPODIVM, 2008.

STUMM, Raquel Denize. Princípio da Proporcionalidade no Direito Constitucional Brasileiro. Porto Alegre : Livraria do Advogado, 1995.

TANAKA, Oswaldo Yoshimi. A Judicialização da Prescrição Medicamentosa no SUS ou o Desafio de Garantir o Direito Constitucional de Acesso à Assistência Farmacêutica. Revista de Direito Sanitário, São Paulo; 9(1):137-143, mar./jun. 2008. [on line] Disponível em http://periodicos.usp.br/index.php/rdisan/article/viewFile/13109/14912, acessado em 10 jul. 2013 